\title{
Parametric resonance in the dynamics of an elliptic vortex in a periodically strained environment
}

\author{
Konstantin V. Koshel ${ }^{1,2,3}$ and Eugene A. Ryzhov ${ }^{1}$ \\ ${ }^{1}$ V.I.Il'ichev Pacific Oceanological Institute, FEB RAS, 43, Baltiyskaya Street, Vladivostok, 690041, Russia \\ ${ }^{2}$ Far Eastern Federal University, 8, Sukhanova Street, Vladivostok, 690950, Russia \\ ${ }^{3}$ Institute of Applied Mathematics, FEB RAS, 7, Radio Street, Vladivostok, 690022, Russia \\ Correspondence to: Konstantin V. Koshel (kvkoshel@poi.dvo.ru)
}

Received: 12 September 2016 - Published in Nonlin. Processes Geophys. Discuss.: 4 October 2016

Revised: 2 December 2016 - Accepted: 21 December 2016 - Published: 12 January 2017

\begin{abstract}
The model of an elliptic vortex evolving in a periodically strained background flow is studied in order to establish the possible unbounded regimes. Depending on the parameters of the exterior flow, there are three classical regimes of the elliptic vortex motion under constant linear deformation: (i) rotation, (ii) nutation, and (iii) infinite elongation. The phase portrait for the vortex dynamics features critical points which correspond to the stationary vortex not changing its form and orientation. We demonstrate that, given superimposed periodic oscillations to the exterior deformation, the phase space region corresponding to the elliptic critical point experiences parametric instability leading to locally unbounded dynamics of the vortex. This dynamics manifests itself as the vortex nutates along the strain axis while continuously elongating. This motion continues until nonlinear effects intervene near the region associated with the steadystate separatrix. Next, we show that, for specific values of the perturbation parameters, the parametric instability is effectively suppressed by nonlinearity in the primal parametric instability zone. The secondary zone of the parametric instability, on the contrary, produces an effective growth of the vortex's aspect ratio.
\end{abstract}

\section{Introduction}

Simplified vortex models have been extremely useful in helping us understand the intricate behaviour of real coherent structures in the ocean and atmosphere. Such models are usually highly nonlinear, making it possible to gain insight into many phenomena that are difficult to predict within a geophysical setting (Provenzale, 1999; Balasuriya and Jones, 2001; Koshel and Prants, 2006; Samelson, 2013; Ryzhov and Koshel, 2013; Kostrykin et al., 2006; Koshel et al., 2008, 2013, 2014; Haller, 2015). For instance, such vortex models can shed some light on the dynamics of interacting coherent mesoscale vortices (Reznik and Dewar, 1994; Gryanik et al., 2000; Reznik and Kizner, 2010; Carton et al., 2010, 2013; Reinaud and Carton, 2015), sustainability of such vortices against external flows (McKiver and Dritschel, 2003; Liu and Roebber, 2008; Perrot and Carton, 2010), or topographic influence (Kozlov et al., 2005; Johnson and McDonald, 2005; Ryzhov et al., 2010; Sutyrin et al., 2011; Nilawar et al., 2012), and so on.

One of the most renowned vortex models is the model of an elliptic vortex subjected to linear deformation consisting of shear and rotational components (Kida, 1981). In the case of a stationary deformation, the elliptic vortex is able to perform three types of motion (Kida, 1981) depending on the parameters of the deformation flow and the initial alignment of the ellipse against the exterior strain. There are two periodic states involving the vortex changing its eccentricity; these are (i) rotation and (ii) nutation. Moreover, there is one aperiodic state - infinite elongation. In this case, the vortex elongates continuously tending to be collinear with the strain axis. Moreover, for a specific initial alignment, the vortex can be stationary.

The model of an elliptic vortex embedded in a linear deformation field is the base model to assess the stability of elliptic vortex shapes occurring in nature. A large body of literature is devoted to the problem. Most of the papers consider spatial perturbations to the elliptic form in the case of con- 
stant linear deformation (Neu, 1984; Melander et al., 1986; Neu, 1990; Dritschel, 1990; Meacham et al., 1990; Legras and Dritschel, 1991; Kida and Takaoka, 1994; Miyazaki and Hanazaki, 1994; Bayly et al., 1996; Meacham et al., 1997; Mitchell and Rossi, 2008). A prominent result of this stability analysis is that an elliptic vortex is stable to linear perturbations of its form until its geometrical shape complies with the relation $a / b \leq 3$, where $a$ and $b$ are the major and minor semi-axes of the ellipse.

Another interesting aspect of the dynamics of an elliptic vortex is its response to time-dependent external deformation. In this case, the form of the vortex endures no changes but the vortex's aspect ratio and orientation can alter unpredictably demonstrating chaotic behavior. This problem has been addressed for small-amplitude strain oscillations by means of the Melnikov's integral technique in several works (Bertozzi, 1988; Dhanak and Marshall, 1993; Ide and Wiggins, 1995; Goldman and McCann, 2008). The evolution of the vortex embedded in a time-dependent strain with a slowly varying frequency was addressed in Friedland (1999). Dhanak and Marshall (1993) also assess the stability of the stationary configuration of the elliptic vortex; i.e., the vortex's aspect ratio and orientation do not change in time under a constant exterior deformation, given a time-dependent external perturbation. They show that such a stationary configuration of the vortex can be easily destabilized into the nutation or even rotation regimes because of a linear resonance effect. In this paper, we demonstrate that, in the case of relatively small amplitudes of the perturbation, the vortex loses its stationarity by means of parametric instability. In the case of finite amplitudes and optimal frequencies of the perturbation, the dynamics of the vortex is governed strictly by nonlinear effects.

It is also worth noting that fluid particle advection near an oscillating elliptic vortex embedded in a constant deformation flow manifests chaotic dynamics (Polvani and Wisdom, 1990; Polvani et al., 1990; Dahleh, 1992). This is because the oscillating elliptic vortex generates a time-periodic perturbation to the fluid particle motion. This results in the appearance of exponentially diverging trajectories in the unsteady velocity field governing the fluid particle advection. Similar chaotic dynamics is present in the case of the ellipsoid vortex model (Zhmur et al., 2011; Koshel et al., 2013, 2015), which is a generalization of the elliptic vortex model taking into account a linear vertical stratification (Zhmur and Pankratov, 1989; Meacham et al., 1994; Dritschel, 2011; McKiver, 2015; McKiver and Dritschel, 2016).

Let us consider an inviscid, incompressible, twodimensional flow. In this flow, an elliptic patch of constant vorticity $g$, experiencing deformation from time-dependent strain $e(t)$ and background rotation $\gamma(t)$, is embedded. The patch conserves its elliptic form with $a$ and $b$ being the ellipse's semi-axes, $\varepsilon=a / b$ being the aspect ratio, and $\varphi$ being the angle between the ellipse's major semi-axis and the $x$ axis of the Cartesian coordinate frame. The governing equations
(Kida, 1981) are

$\dot{\varepsilon}=2 e \varepsilon \cos 2 \varphi, \quad \dot{\varphi}=\gamma+\frac{g \varepsilon}{(\varepsilon+1)^{2}}-e \frac{\varepsilon^{2}+1}{\varepsilon^{2}-1} \sin 2 \varphi$.

It is worth noticing that the motion of the ellipse's center $\left(x_{0}, y_{0}\right)$ is governed by advection equations in the form

$\frac{\mathrm{d} x_{0}}{\mathrm{~d} t}=u_{0}+e\left(x_{0}-x_{\mathrm{d}}\right)-\gamma\left(y_{0}-y_{\mathrm{d}}\right)$,
$\frac{\mathrm{d} y_{0}}{\mathrm{~d} t}=v_{0}-e\left(y_{0}-y_{\mathrm{d}}\right)+\gamma\left(x_{0}-x_{\mathrm{d}}\right)$,

where $u_{0}, v_{0}$ are the velocity's components of an arbitrary uniform flow; $x_{\mathrm{d}}, y_{\mathrm{d}}$ are the coordinates of the constant deformation center. Equation (2) exactly coincides with the equations governing the motion of the vorticity center of an arbitrary number of point vortices embedded in such a deformation flow (Koshel and Ryzhov, 2012; Ryzhov and Koshel, 2016). Given periodic unequal dependencies of the strain and rotation components, these equations allow for parametric instability. This, in turn, may result in unbounded motion of the vorticity center depending on the strain and rotation oscillation parameters. However, the relative vortex motion is independent of the vortex's center motion. We further consider the evolution of the elliptic vortex when the vortex's center and the exterior deformation's center coincide at the coordinate's origin.

Without loss of generality, the ellipse's vorticity in Eq. (1) is further set to be $g=1$. Equation (1) represent a dynamical system with "one and a half" degrees of freedom (Lichtenberg and Lieberman, 1983; Zaslavsky, 1998) given timedependent strain and rotation rates $e(t)$ and $\gamma(t)$. This suggests that the dynamics of the phase variables may be chaotic for certain initial conditions. To start, it is informative to look into the stationary system given constant values of $e(t) \equiv$ $e_{0}=$ const, $\gamma(t) \equiv \gamma_{0}=$ const. The critical points $\left(\varphi_{0}, \varepsilon_{0}\right)$ corresponding to the stationary elliptic vortex ensue from the following relations (Kida, 1981; Bayly et al., 1996):

$$
\begin{aligned}
& \varphi_{0}= \pm \frac{\pi}{4}, \\
& \varepsilon_{0}^{3}+\varepsilon_{0}^{2} \frac{\left(\gamma_{0}-e_{0} \sin 2 \varphi_{0}+1\right)}{\left(\gamma_{0}-e_{0} \sin 2 \varphi_{0}\right)}-\varepsilon_{0} \frac{\left(\gamma_{0}+e_{0} \sin 2 \varphi_{0}+1\right)}{\left(\gamma_{0}-e_{0} \sin 2 \varphi_{0}\right)} \\
& -\frac{\left(\gamma_{0}+e_{0} \sin 2 \varphi_{0}\right)}{\left(\gamma_{0}-e_{0} \sin 2 \varphi_{0}\right)}=0 .
\end{aligned}
$$

There are four qualitatively different phase portraits depending on the number and type of the critical points (Bayly et al., 1996). Each phase portrait has at least one elliptic critical point. The corresponding phase portrait for the values $e_{0}=0.15, \gamma_{0}=0.02$ is shown in Fig. 1 . The homoclinic separatrix delineates the regions of the initial conditions corresponding to the ellipse nutation (near the elliptic critical point) and infinite elongation (all the rest).

The elliptical critical point illustrated in Fig. 1 corresponds to the elliptic vortex, without performing any motion. Once 


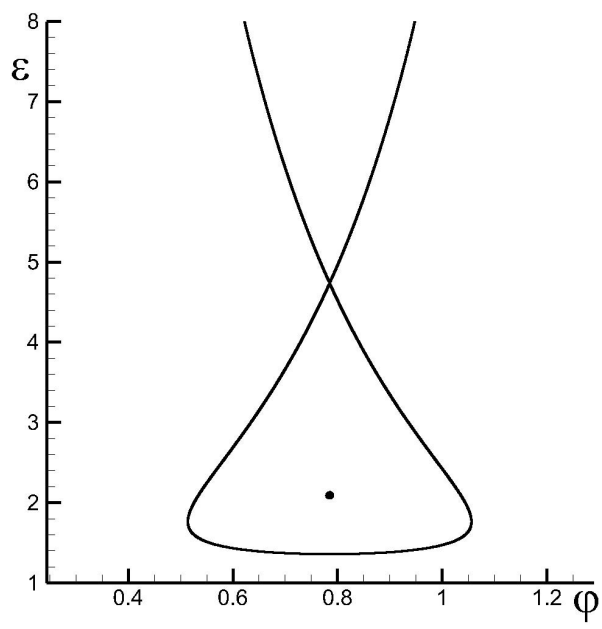

Figure 1. The phase portrait of the stationary system (1) in the case of one elliptic and one hyperbolic critical point for $e_{0}=0.15, \gamma_{0}=$ 0.02 .

slightly shifted from this stance, the vortex starts nutating, slightly changing the lengths of its semi-axes and the orientation angle. If an initial condition is taken outside of the nutation region near the elliptic critical point, the vortex starts elongating infinitely.

\section{The dynamics of the perturbed system near the stationary position}

Now, let us examine the dynamics of the system near the elliptic critical point, given small time-dependent perturbations of the deformation flow

$$
\begin{gathered}
e(t)=e_{0}+e^{\prime}(t)=e_{0}+e_{0} \delta \cos v t, \\
\gamma(t)=\gamma_{0}+\gamma^{\prime}(t)=\gamma_{0}+\gamma_{0} \delta \cos v t .
\end{gathered}
$$

One can expand Eq. (1) into a Taylor series up to the first term over a small deviation from the elliptic critical point

$\varepsilon(t) \approx \varepsilon_{0}+\varepsilon^{\prime}(t), \quad \varphi(t) \approx \varphi_{0}+\varphi^{\prime}(t)= \pm \frac{\pi}{4}+\varphi^{\prime}(t)$,

where $\varepsilon_{0}, \varphi_{0}$ result from Eq. (3); $\varepsilon^{\prime}(t), \varphi^{\prime}(t)$ are small timedependent deviations. One then obtains the following for the deviations:

$$
\begin{aligned}
\frac{\mathrm{d} \varepsilon^{\prime}}{\mathrm{d} t} & =-4 e(t) \varepsilon_{0} \varphi^{\prime} \sin 2 \varphi_{0}, \\
\frac{\mathrm{d} \varphi^{\prime}}{\mathrm{d} t} & =\left[\gamma(t)-e(t) \frac{\varepsilon_{0}^{2}+1}{\varepsilon_{0}^{2}-1} \sin 2 \varphi_{0}\right] \\
& -\varepsilon^{\prime}\left[\frac{\left(\varepsilon_{0}-1\right)}{\left(\varepsilon_{0}+1\right)^{3}}-4 e(t) \frac{\varepsilon_{0}}{\left(\varepsilon_{0}^{2}-1\right)^{2}} \sin 2 \varphi_{0}\right] .
\end{aligned}
$$

The linear system of Eq. (6) is an inhomogeneous one. However, in terms of determining whether the system has unbounded solutions, one can take advantage of the associated homogeneous system:

$$
\begin{aligned}
\frac{\mathrm{d} \varepsilon^{\prime}}{\mathrm{d} t} & =-4 e(t) \varepsilon_{0} \varphi^{\prime} \sin 2 \varphi_{0}, \\
\frac{\mathrm{d} \varphi^{\prime}}{\mathrm{d} t} & =-\varepsilon^{\prime}\left[\frac{\left(\varepsilon_{0}-1\right)}{\left(\varepsilon_{0}+1\right)^{3}}-4 e(t) \frac{\varepsilon_{0}}{\left(\varepsilon_{0}^{2}-1\right)^{2}} \sin 2 \varphi_{0}\right] .
\end{aligned}
$$

It is worth noting that the term with the background rotation $\gamma$ has vanished from the homogeneous system. This readily means that the existence of unbounded solutions about the stationary position is independent of the exterior rotation.

One can see that, if reduced to a second-order equation, the system of Eq. (7) represents a Hill equation (Magnus and Winkler, 1966). This, in turn, signifies that there is a possible manifestation of parametric instability near the vortex stationary position. In other words, given specific values of the perturbation's parameters, the phase trajectories become unbounded near the steady-state elliptic critical point. The values of the perturbation parameters resulting in parametric instability can be readily figured out using the Floquet analysis. An analytical estimate can be derived by means of averaging techniques (Klyatskin and Koshel, 1983; Koshel and Ryzhov, 2012, 2016; Ryzhov and Koshel, 2016). To do this, it is convenient to rewrite the system of Eq. (7) in the form

$$
\begin{aligned}
\frac{\mathrm{d} \varepsilon^{\prime}}{\mathrm{d} t} & =-4 e(t) \varepsilon_{0} \varphi^{\prime} \sin 2 \varphi_{0}, \\
\frac{\mathrm{d} \varphi^{\prime}}{\mathrm{d} t} & =\left(\frac{k^{2}}{4 e_{0} \varepsilon_{0} \sin 2 \varphi_{0}}+4 e_{0} \frac{\varepsilon_{0} \sin 2 \varphi_{0}}{\left(\varepsilon_{0}^{2}-1\right)^{2}} \delta \cos v t\right) \varepsilon^{\prime},
\end{aligned}
$$

where $k^{2}=-\frac{4 e_{0} \varepsilon_{0} \sin 2 \varphi_{0}}{\left(\varepsilon_{0}+1\right)^{2}}\left[\frac{\left(\varepsilon_{0}-1\right)}{\left(\varepsilon_{0}+1\right)}-4 \frac{e_{0} \varepsilon_{0}}{\left(\varepsilon_{0}-1\right)^{2}} \sin 2 \varphi_{0}\right]$.

Let us introduce a new variable, $\rho=$ $-\left(1+i \frac{4 e_{0} \varepsilon_{0} \sin 2 \varphi_{0}}{k} \frac{\varphi^{\prime}}{\varepsilon^{\prime}}\right) e^{-i v t}$. Then, instead of Eq. (8), one obtains

$$
\begin{aligned}
\frac{\mathrm{d} \rho}{\mathrm{d} t} & =i\left(2 k\left[1+\frac{\delta\left(e^{i v t}+e^{-i v t}\right)}{2 e_{0}}\right]-v\right) \rho \\
& -i k\left[\frac{16}{k^{2}} \frac{e_{0}^{2} \varepsilon_{0}^{2}}{\left(\varepsilon_{0}^{2}-1\right)^{2}}-1\right] \frac{\delta\left(1+e^{-2 i v t}\right)}{2 e_{0}} \\
& +i k\left[e^{i v t}+\frac{\delta\left(e^{2 i v t}+1\right)}{2 e_{0}}\right] \rho^{2} .
\end{aligned}
$$

Then, taking into account that the fast-oscillating terms average to zero, one gets the following for the averaged value 


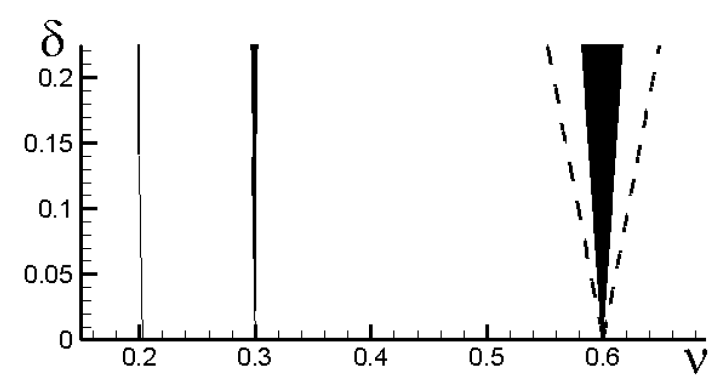

Figure 2. The regions of parametric instability in the parametric plane $(v, \delta)$ for $e_{0}=0.15, \gamma_{0}=0.02$. The dark regions correspond to locally unbounded dynamics of the ellipse obtained with the use of the linear approximation (Eq. 7). The dashed lines mark the region incurred from the analytical estimate (Eq. 12).

$$
\begin{aligned}
& \bar{\rho}: \\
& \frac{\mathrm{d} \bar{\rho}}{\mathrm{d} t}=i k \frac{\delta}{2 e_{0}} \bar{\rho}^{2}+i(2 k-v) \bar{\rho} \\
& -i k\left[\frac{16}{k^{2}} \frac{e_{0}^{2} \varepsilon_{0}^{2}}{\left(\varepsilon_{0}^{2}-1\right)^{2}}-1\right] \frac{\delta}{2 e_{0}}, \bar{\rho}(0)=0 .
\end{aligned}
$$

The solution of Eq. (10) is

$$
\frac{\left(\bar{\rho}+\frac{e_{0}}{\delta k}(2 k-v)-D\right)}{\left(\bar{\rho}+\frac{e_{0}}{\delta k}(2 k-v)+D\right)}=\exp \left\{i k \frac{D \delta}{e_{0}}\right\}
$$

where $D^{2}=\left(\frac{e_{0}}{\delta k}(2 k-v)\right)^{2}-\left[\frac{16}{k^{2}} \frac{e_{0}^{2} \varepsilon_{0}{ }^{2}}{\left(\varepsilon_{0}+1\right)^{2}\left(\varepsilon_{0}-1\right)^{2}}-1\right]$.

The solution Eq. (11) grows unboundedly if the argument in the right-hand term exponential function is real. Therefore, a rough analytical estimate for the location of the primary parametric instability zone in the parametric space ensues

$$
(2 k-v)= \pm 2 \delta \sqrt{\frac{\sin 2 \varphi_{0}}{e_{0}} \frac{\varepsilon_{0}\left(\varepsilon_{0}-1\right)}{\left(\varepsilon_{0}+1\right)^{3}}} .
$$

Figure 2 depicts the precise parametric instability zones obtained by the Floquet analysis (the dark regions) and the analytically estimated values (the dashed line) in the $(\delta, v)$ parametric space. Every parameter set from the dark regions leads to spiral-like unbounded trajectories of the linear system of Eq. (7), while the parameters taken from the light areas result in periodic trajectories in bounded regions. The primary zone of the parametric instability is the widest zone located near $v=0.6$. The secondary zone is the one located near $v=0.3$.

\section{Nonlinear suppression of the solution growth attributed to the linear parametric instability}

Now, let us discuss possible implications of the parametric instability reported for the linear system of Eq. (7) that governs the dynamics of the elliptic vortex in the immediate vicinity of the elliptical critical point shown in Fig. 1. Since the original system of Eq. (1) is nonlinear, it is clear that the applicability range of the linear system of Eq. (7) is relatively limited.

First of all, in the case of parametric instability, the trajectories that originated near the steady-state elliptic critical point move unboundedly only up to the steady-state separatrix region where nonlinear effects prevail against the linear unbounded motion. This scenario occurs only if the nonlinear effects do not already drastically influence the dynamics in the immediate vicinity of the steady-state critical elliptic point. This is the case, for instance, for the primary parametric instability zone shown in Fig. 2. Given the corresponding perturbation values, the phase space near the steady-state critical elliptical point differs crucially from the unperturbed phase space.

To corroborate this effect, a Poincaré section shown in Fig. 3a is presented. To construct this and all the following Poincaré sections, we plot the position of a phase trajectory exactly in a perturbation period $2 \pi / \nu$. Thus, a chaotic trajectory appears as a set of disorder points and a regular trajectory appears as a smooth, closed, linked orbit in the sections. Figure $3 \mathrm{a}$ illustrates that the dynamics near the steady-state elliptic critical point is dominated by a nonlinear resonance with winding number $1: 2$. Because of this effect, the phase trajectories that originated near the steady-state elliptic critical point do not demonstrate parametric instability. Figure $3 b$ shows a trajectory starting at the steady-state elliptic critical point $\varphi_{0}=\pi / 4, \varepsilon_{0} \approx 2.09244$ is clearly bounded to the region of the nonlinear resonance influence. Therefore, the linear system (Eq. 7) cannot account for the dynamics in this case.

Nevertheless, the linear system is a good approximation for certain values of the perturbation's parameters. For instance, when one considers the second parametric instability zone, the perturbed phase space near the steady-state elliptic critical point features no nonlinear resonances (see the Poincaré section shown in Fig. 4a). Therefore, the dynamics near the steady-state critical elliptic point can be derived from the linear system (Eq. 7). When this happens, the phase trajectory that starts near the steady-state critical elliptic point moves in a spiral-like divergent trajectory (see Fig. 4b). The trajectory spirals only until it reaches the region of high nonlinearity (a chaotic region in the place of the Poincaré section in Fig. 4a); then it spirals back. However, the parametric resonance results in a significant change of the ellipse characteristics contrary to the case shown in Fig. 3b.

\section{Conclusions}

In this work, we have considered a model of an isolated elliptic vortex embedded in a time-dependent deformation flow, consisting of strain and rotational components. The 

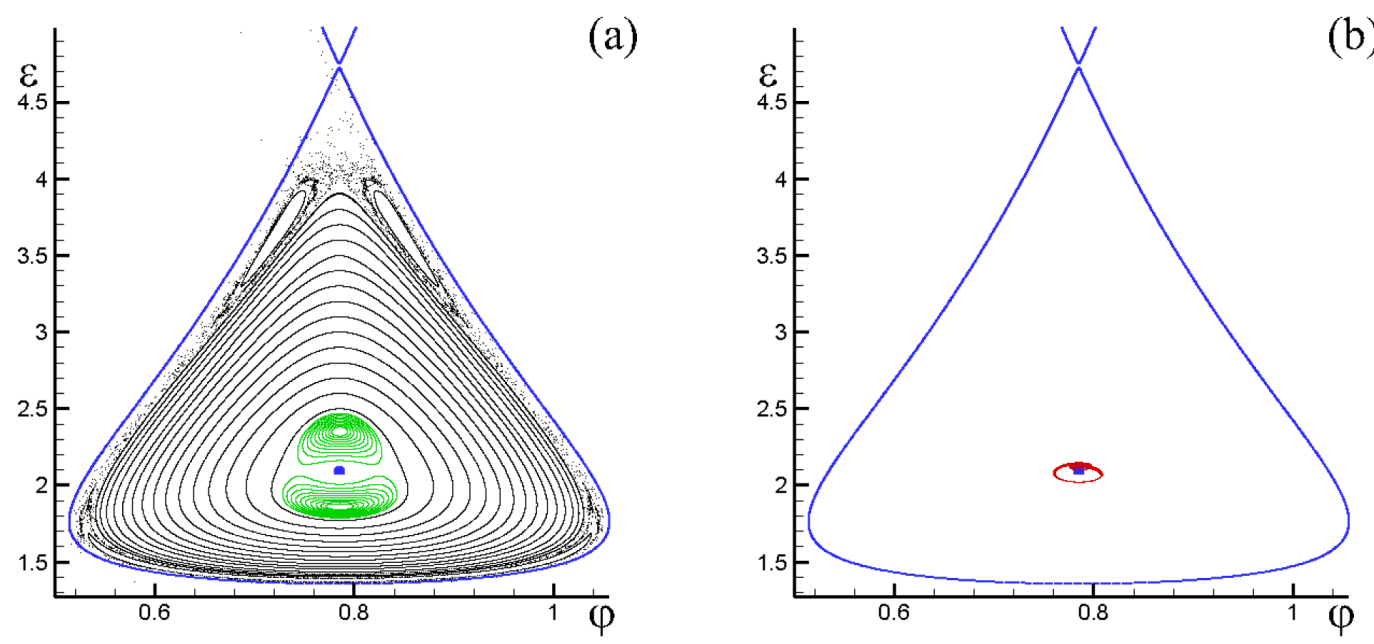

(b)

Figure 3. The dynamics of the perturbed system in the case of the primary parametric instability zone of the corresponding linearized system (Eq. 7) for $e_{0}=0.15, \gamma_{0}=0.02$ and perturbation parameters $\delta=0.01, v=0.6$. The solid blue lines show the steady-state separatrix. The blue dot shows the steady-state elliptic critical point $\varphi_{0}=\pi / 4, \varepsilon_{0} \approx 2.09244$. Panel (a) shows a Poincaré section illustrating the appearance of a highly nonlinear zone (the green orbits) near the steady-state elliptic critical point that prohibits the divergent motion due to the linear parametric resonance; (b) a phase trajectory starting at the steady-state elliptic critical point bounded to the region of the nonlinear resonance, illustrating a vanishing effect of the linear parametric resonance.
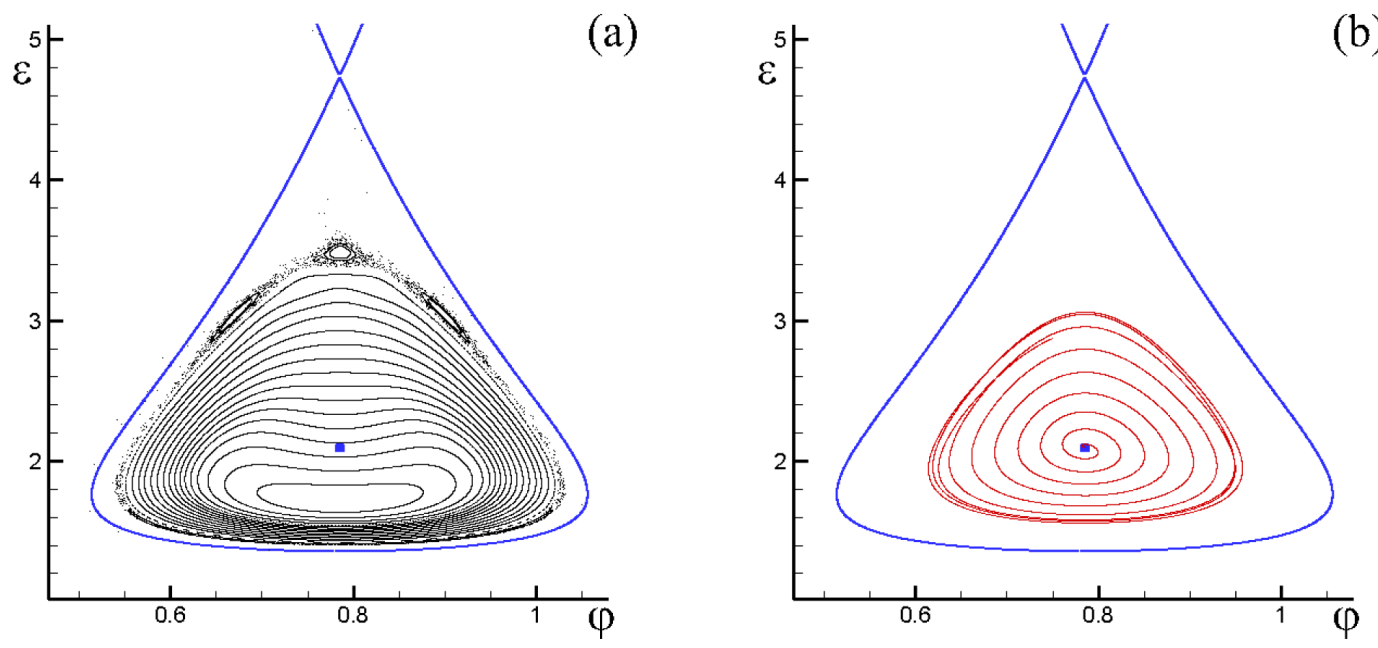

Figure 4. The same as in Fig. 3, except $v=0.3$. Panel (a) shows a Poincaré section illustrating that the perturbed system remains largely linear near the steady-state elliptic critical point; (b) a divergent spiralling phase trajectory starting at the steady-state elliptic critical point experiencing linear parametric instability.

main focus is on the dynamics of the vortex near its steadystate stable position. In the linear approximation, the vortex dynamics is established to experience parametric instability. This instability entails the following motion of the vortex. The vortex starts nutating about the strain axis, continuously increasing the aspect ratio of its semi-axes. This evolution continues until the phase trajectory reaches the region of high nonlinearity near the steady-state separatrix. This regime proceeds, provided the nonlinear system governing the dynamics of the vortex remains relatively linear near the steady-state elliptic point under perturbation. We have shown that this, in fact, is possible for certain values of the perturbation parameters. Thus, our results suggest that the parametric instability, which is intrinsic to linear systems, can also be an important factor in the nonlinear system in question.

However, the influence of linear effects on the nonlinear systems should be generally interpreted with great care. Indeed, the primary parametric resonance zone produces the most effective unbounded motion of the phase trajectories in the linearized system, but the same driving parameters applied to the nonlinear system lead only to a bounded motion in a very small vicinity of the steady-state elliptic critical 
point. This is because a region of high nonlinearity appears near the steady-state elliptic critical point that completely suppresses the linear unbounded motion in the nonlinear system.

Considering the evolution of the elliptic vortex, the elliptic vortex nutates along the shear axis, becoming more oblong in time. This dynamics is regular (not chaotic), meaning that the vortex form returns to its initial shape after the locally unbounded motion is suppressed by the nonlinear effects near the steady-state separatrix. However, during this temporal elongation, the aspect ratio of the vortex may easily exceed the critical value of the vortex stability. Thus, in terms of the linear stability of the vortex to the perturbations of its elliptic form, the vortex turns unstable. Therefore, our results may suggest the following scenario of the evolution of an elliptic vortex in a time-dependent natural environment. First, it starts becoming more and more oblate due to the linear parametric instability. After attaining the critical aspect ratio, small-scale disturbances start transpiring on the boundary of the vortex. Finally, it may break into a number of smaller elliptic vortices (Carton et al., 1989; Polvani and Carton, 1990; Carton and Legras, 1994).

\section{Data availability}

No specific data are used. All the figures are obtained by numerically integrated equations (Eq. 1) using a standard Bulirsch-Stoer procedure, which uses the Richardson extrapolation and modified midpoint method.

Acknowledgements. The publication of this paper is made possible thanks to the Office of Naval Research (grant no. N00014-16-12492). The work of EAR in calculating the parameters leading to instability was partially supported by the Ministry of Education and Science of Russian Federation (project no. MK-172.2017.1) and by the Russian Foundation for Basic Research (project no. 15-05-00103). The work of KVK in obtaining the analytical estimate was supported by the Russian Scientific Foundation (project no. 16-17-10025).

Edited by: A. M. Mancho

Reviewed by: three anonymous referees

\section{References}

Balasuriya, S. and Jones, C. K. R. T.: Diffusive draining and growth of eddies, Nonlin. Processes Geophys., 8, 241-251, doi:10.5194/npg-8-241-2001, 2001.

Bayly, B. J., Holm, D. D., and Lifschitz, A.: Three-dimensional stability of elliptical vortex columns in external strain flows, Philos. T. Roy. Soc. A, 354, 895-926, doi:10.1098/rsta.1996.0036, 1996.

Bertozzi, A. L.: Heteroclinic orbits and chaotic dynamics in planar fluid flows, SIAM J. Math. Anal., 19, 1271-1294, doi:10.1137/0519093, 1988.
Carton, X. and Legras, B.: The life-cycle of tripoles in 2dimensional incompressible flows, J. Fluid Mech., 267, 53-82, doi:10.1017/S0022112094001114, 1994.

Carton, X., Meunier, T., Flierl, G. R., Perrot, X., and Sokolovskiy, M.: Explosive instability of geostrophic vortices, Part 2: parametric instability, Theor. Comput. Fluid Dyn., 24, 131-135, doi:10.1007/s00162-009-0139-0, 2010.

Carton, X., Le Cann, B., Serpette, A., and Dubert, J.: Interactions of surface and deep anticyclonic eddies in the Bay of Biscay, J. Mar. Syst., 109, S45-S59, doi:10.1016/j.jmarsys.2011.09.014, 2013.

Carton, X. J., Flierl, G. R., and Polvani, L. M.: The generation of tripoles from unstable axisymmetric isolated vortex structures, Europhys. Lett., 9, 339-334, doi:10.1209/0295-5075/9/4/007, 1989.

Dahleh, M. D.: Exterior flow of the Kida ellipse, Phys. Fluids A, 4, 1979-1985, doi:10.1063/1.858366, 1992.

Dhanak, M. R. and Marshall, M. P.: Motion of an elliptic vortex under applied periodic strain, Phys. Fluids, 5, 1224-1230, doi:10.1063/1.858608, 1993.

Dritschel, D. G.: The stability of elliptical vortices in an external straining flow, J. Fluid Mech., 210, 223-261, doi:10.1017/S0022112090001276, 1990.

Dritschel, D. G.: An exact steadily rotating surface quasigeostrophic elliptical vortex, Geophys. Astrophys. Fluid Dyn., 4-5, 368-376, doi:10.1080/03091929.2010.485997, 2011.

Friedland, L.: Control of Kirchhoff vortices by a resonant strain, Phys. Rev. E, 59, 4106, doi:10.1103/PhysRevE.59.4106, 1999.

Goldman, D. and McCann, R. J.: Chaotic response of the 2D semigeostrophic and 3D quasi-geostrophic equations to gentle periodic forcing, Nonlinearity, 21, 1455-1470, doi:10.1088/09517715/21/7/005, 2008.

Gryanik, V. M., Doronina, T. N., Olbers, D. J., and Warncke, T. H.: The theory of three-dimensional hetons and vortexdominated spreading in localized turbulent convection in a fast rotating stratified fluid, J. Fluid Mech., 423, 71-125, doi:10.1017/S002211200000183X, 2000.

Haller, G.: Lagrangian coherent structures, Annuv. Rev. Fluid Mech., 47, 137-162, doi:10.1146/annurev-fluid-010313-141322, 2015.

Ide, K. and Wiggins, S.: The dynamics of elliptically shaped regions of uniform vorticity in time-periodic, linear external velocity fields, Fluid Dyn. Res., 15, 205-235, doi:10.1016/01695983(95)94956-T, 1995.

Johnson, E. and McDonald, N.: The point island approximation in vortex dynamics, Geophys. Astrophys. Fluid Dyn., 99, 49-60, doi:10.1080/03091920512331323896, 2005.

Kida, S.: Motion of an elliptic vortex in a uniform shear flow, J. Phys. Soc. Jpn., 50, 3517-3520, doi:10.1143/JPSJ.50.3517, 1981.

Kida, S. and Takaoka, M.: Vortex reconnection, Annu. Rev. Fluid Mech., 26, 169-189, doi:10.1146/annurev.fluid.26.1.169, 1994.

Klyatskin, V. I. and Koshel, K. V.: Numerical modeling of wave propagation in periodic media, J. Exp. Theor. Phys., 84, 20922098, 1983.

Koshel, K. V. and Prants, S. V.: Chaotic advection in the ocean, Physics-Uspekhi, 49, 1151-1178, doi:10.1070/PU2006v049nl1ABEH006066, 2006. 
Koshel, K. V. and Ryzhov, E. A.: Parametric resonance with a pointvortex pair in a nonstationary deformation flow, Phys. Lett. A, 376, 744-747, doi:10.1016/j.physleta.2011.12.016, 2012.

Koshel, K. V. and Ryzhov, E. A.: Local parametric instability near elliptic points in vortex flows under shear deformation, Chaos, 26, 083111, doi:10.1063/1.4961123, 2016.

Koshel, K. V., Sokolovskiy, M. A., and Davies, P. A.: Chaotic advection and nonlinear resonances in an oceanic flow above submerged obstacle, Fluid Dyn. Res., 40, 695-736, doi:10.1016/j.fluiddyn.2008.03.001, 2008.

Koshel, K. V., Ryzhov, E. A., and Zhmur, V. V.: Diffusion-affected passive scalar transport in an ellipsoidal vortex in a shear flow, Nonlin. Processes Geophys., 20, 437-444, doi:10.5194/npg-20437-2013, 2013.

Koshel, K. V., Ryzhov, E. A., and Zyryanov, V. N.: Toroidal vortices over isolated topography in geophysical flows, Fluid Dyn. Res., 46, 031405, doi:10.1088/0169-5983/46/3/031405, 2014.

Koshel, K. V., Ryzhov, E. A., and Zhmur, V. V.: Effect of the vertical component of diffusion on passive scalar transport in an isolated vortex model, Phys. Rev. E, 92, 053021, doi:10.1103/PhysRevE.92.053021, 2015.

Kostrykin, S. V., Khapaev, A. A., Ponomarev, V. M., and Yakushkin, I. G.: Lagrangian structures in time-periodic vortical flows, Nonlin. Processes Geophys., 13, 621-628, doi:10.5194/npg-13-6212006, 2006.

Kozlov, V. F., Koshel, K. V., and Stepanov, D. V.: Influence of the boundary on chaotic advection in the simplest model of a topographic vortex, Izv. Atmos. Ocean. Phys., 41, 217-227, 2005.

Legras, B. and Dritschel, D.: The elliptical model of twodimensional vortex dynamics, I: The basic state, Phys. Fluids, 3, 845-854, doi:10.1063/1.858015, 1991.

Lichtenberg, A. and Lieberman, M.: Regular and Stochastic Motion, Springer-Verlag, New York, 1983.

Liu, Z. and Roebber, P. J.: Vortex-Driven Sensitivity in Deformation Flow, J. Phys. Oceanogr., 65, 3819-3839, doi:10.1175/2008JAS2745.1, 2008.

Magnus, W. and Winkler, S.: Hill's equation, Interscience-Wiley, 1966.

McKiver, W. J.: The Ellipsoidal Vortex: A Novel Approach to Geophysical Turbulence, Adv. Math. Phys., 2015, 613683, doi:10.1155/2015/613683, 2015.

McKiver, W. J. and Dritschel, D.: Balanced solutions for an ellipsoidal vortex in a rotating stratified flow, J. Fluid Mech., 802, 333-358, doi:10.1017/jfm.2016.462, 2016.

McKiver, W. J. and Dritschel, D. G.: The motion of a fluid ellipsoid in a general linear background flow, J. Fluid Mech., 474, 147173, doi:10.1017/S0022112002002859, 2003.

Meacham, S. P., Flierl, G. R., and Send, U.: Vortices in shear, Dynam. Atmos. Oceans, 14, 333-386, 1990.

Meacham, S. P., Pankratov, K. K., Shchepetkin, A. F., and Zhmur, V. V.: The interaction of ellipsoidal vortices with background shear flows in a stratified fluid, Dynam. Atmos. Oceans, 21, 167212, doi:10.1016/0377-0265(94)90008-6, 1994.

Meacham, S. P., Morrison, P. J., and Flierl, G. R.: Hamiltonian moment reduction for describing vortices in shear, Phys. Fluids, 9, 2310-2328, doi:10.1063/1.869352, 1997.

Melander, M. V., Zabusky, N. J., and S., S. A.: A moment model for vortex interactions of the two-dimensional Euler equations. Part 1. Computational validation of a Hamil- tonian elliptical representation, J. Fluid Mech., 167, 95-115, doi:10.1017/S0022112086002744, 1986.

Mitchell, T. B. and Rossi, L. F.: The evolution of Kirchhoff elliptic vortices, Phys. Fluids, 20, 054103, doi:10.1063/1.2912991, 2008.

Miyazaki, T. and Hanazaki, H.: Baroclinic instability of Kirchhoff's elliptic vortex, J. Fluid Mech., 261, 253-271, doi:10.1017/S0022112094000339, 1994.

Neu, J. C.: The dynamics of a columnar vortex in an imposed strain, Phys. Fluids, 27, 2397-2402, doi:10.1063/1.864543, 1984.

Neu, J. C.: Vortices in complex scalar fields, Physica D, 43, 385406, doi:10.1016/0167-2789(90)90143-D, 1990.

Nilawar, R., Johnson, E., and McDonald, N.: Finite Rossby radius effects on vortex motion near a gap, Phys. Fluids, 24, 066601, doi:10.1063/1.4721432, 2012.

Perrot, X. and Carton, X.: 2D vortex interaction in a nonuniform flow, Theor. Comput. Fluid Dyn., 24, 95-100, doi:10.1007/s00162-009-0127-4, 2010.

Polvani, L. M. and Carton, X. J.: The tripole - a new coherent vortex structure of incompressible 2-dimensional flows, Geophys. Astrophys. Fluid Dyn., 51, 87-102, doi:10.1080/03091929008219852, 1990.

Polvani, L. M. and Wisdom, J.: Chaotic Lagrangian trajectories around an elliptical vortex patch embedded in a constant and uniform background shear flow, Phys. Fluids A, 2, 123-126, doi:10.1063/1.857814, 1990.

Polvani, L. M., Wisdom, J., DeJong, E., and Ingersoll, A. P.: Simple Dynamical Models of Neptune's Great Dark Spot, Science, 249, 1393-1398, doi:10.1126/science.249.4975.1393, 1990.

Provenzale, A.: Transport by coherent barotropic vortices, Annu. Rev. Fluid Mech., 31, 55-93, doi:10.1146/annurev.fluid.31.1.55, 1999.

Reinaud, J. N. and Carton, X.: Existence, stability and formation of baroclinic tripoles in quasi-geostrophic flows, J. Fluid Mech., 785, 1-30, doi:10.1017/jfm.2015.614, 2015.

Reznik, G. and Dewar, W.: An analytical theory of distributed axisymmetric barotropic vortices on the beta plane, J. Fluid Mech., 269, 301-321, doi:10.1017/S0022112094001576, 1994.

Reznik, G. M. and Kizner, Z.: Singular vortices in regular flows, Theor. Comput. Fluid Dyn., 24, 65-75, doi:10.1007/s00162-0090150-5, 2010.

Ryzhov, E., Koshel, K., and Stepanov, D.: Background current concept and chaotic advection in an oceanic vortex flow, Theor. Comput. Fluid Dyn., 24, 59-64, doi:10.1007/s00162-009-0170$1,2010$.

Ryzhov, E. A. and Koshel, K. V.: Interaction of a monopole vortex with an isolated topographic feature in a three-layer geophysical flow, Nonlin. Processes Geophys., 20, 107-119, doi:10.5194/npg-20-107-2013, 2013.

Ryzhov, E. A. and Koshel, K. V.: Parametric instability of a many point-vortex system in a multi-layer flow under linear deformation, Regul. Chaotic Dyn., 21, 254-266, doi:10.1134/S1560354716030023, 2016.

Samelson, R. M.: Lagrangian Motion, Coherent Structures, and Lines of Persistent Material Strain, Annu. Rev. Mar. Sci., 5, 11.111.27, doi:10.1146/annurev-marine-120710-100819, 2013.

Sutyrin, G., Herbette, S., and Carton, X.: Deformation and splitting of baroclinic eddies encountering a tall 
seamount, Geophys. Astrophys. Fluid Dyn., 105, 478-505, doi:10.1080/03091929.2011.566566, 2011.

Zaslavsky, G.: Physics of Chaos in Hamiltonian Dynamics, Imperial College Press, London, 1998.

Zhmur, V. V. and Pankratov, K. K.: The dynamics of the semiellipsoid subsurface vortex in the non-uniform flow, Oceanology, 29, 205-211, 1989.
Zhmur, V. V., Ryzhov, E. A., and Koshel, K. V.: Ellipsoidal vortex in a nonuniform flow: Dynamics and chaotic advections, J. Mar. Res., 69, 435-461, doi:10.1357/002224011798765204, 2011. 